\title{
Correction to: Combination of multi-model statistical analysis and quantitative fingerprinting in quality evaluation of Shuang-huang-lian oral liquid
}

\author{
Wei Si ${ }^{1,2} \cdot$ Yali Qiao $^{1,2} \cdot$ Zhe Liu $^{1,2} \cdot$ Gaowa Jin $^{1} \cdot$ Yanfang Liu $^{1} \cdot$ Xingya Xue $^{1} \cdot$ Han Zhou $^{1} \cdot$ Yanming Liu $^{3} \cdot$ Aijin Shen $^{1} \cdot$ \\ Xinmiao Liang ${ }^{1}$
}

Received: 2 September 2020 / Accepted: 2 September 2020 / Published online: 1 October 2020

(C) Springer-Verlag GmbH Germany, part of Springer Nature 2020

\section{Correction to: Anal Bioanal Chem}

https://doi.org/10.1007/s00216-020-02841-z

The authors would like to call the reader's attention to the fact that unfortunately there was a mistake in Table 1 of this contribution. Please find the correct Table 1 below.

Table 1 Calibration curve, linearity, LODs, LOQs, intra-day, inter-day precision, stability, and recovery of three analytes

\begin{tabular}{|c|c|c|c|c|c|c|c|c|c|c|}
\hline \multirow[t]{2}{*}{ Analyte } & \multirow{2}{*}{$\begin{array}{l}\text { Calibration } \\
\text { Curve }\end{array}$} & \multirow{2}{*}{$\begin{array}{l}\text { Linear range } \\
(\mu \mathrm{g} / \mathrm{mL})\end{array}$} & \multirow[t]{2}{*}{$\mathrm{R}^{2}$} & \multirow{2}{*}{$\begin{array}{l}\text { LOD } \\
(\mu \mathrm{g} / \mathrm{mL})\end{array}$} & \multirow{2}{*}{$\begin{array}{l}\text { LOQ } \\
(\mu \mathrm{g} / \mathrm{mL})\end{array}$} & \multicolumn{2}{|c|}{ Precision (RSD \%) } & \multirow{2}{*}{$\begin{array}{l}\text { Stability (RSD \%) } \\
(\mathrm{n}=9)\end{array}$} & \multicolumn{2}{|c|}{ Accuracy $(n=6)$} \\
\hline & & & & & & $\begin{array}{l}\text { Intra-day } \\
(\mathrm{n}=6)\end{array}$ & $\begin{array}{l}\text { Inter-day } \\
(\mathrm{n}=18)\end{array}$ & & $\begin{array}{l}\text { Recovery } \\
(\%)\end{array}$ & $\begin{array}{l}\text { RSD } \\
(\%)\end{array}$ \\
\hline $\begin{array}{l}\text { Chlorogenic } \\
\text { acid }^{1}\end{array}$ & $y=33589 x-24514$ & $6.1 \sim 193.6$ & 0.9999 & 0.3 & 1.5 & 0.8 & 1.8 & 2.6 & $96.0 \sim 97.5$ & 0.5 \\
\hline Phillyrin $^{2}$ & $y=14673 x-1986.9$ & $3.0 \sim 94.9$ & 0.9999 & 0.7 & 2.0 & 0.9 & 2.2 & 2.8 & $102.9 \sim 107.7$ & 1.4 \\
\hline Baicalin $^{1}$ & $y=19345 x-23330$ & $14.6 \sim 467.5$ & 0.9999 & 0.4 & 1.1 & 0.7 & 2.0 & 1.9 & $102.7 \sim 104.0$ & 0.8 \\
\hline
\end{tabular}

Notes: 1: $327 \mathrm{~nm}$ as detection wavelength; $2: 235 \mathrm{~nm}$ as detection wavelength

The online version of the original article can be found at https://doi.org/ $10.1007 / \mathrm{s} 00216-020-02841-\mathrm{z}$

Aijin Shen

ajshen@dicp.ac.cn

$\triangle$ Xinmiao Liang

liangxm@dicp.ac.cn

1 Key Lab of Separation Science for Analytical Chemistry, Dalian Institute of Chemical Physics, Chinese Academy of Sciences, 457 Zhongshan Road, Dalian 116023, Liaoning, China

2 University of Chinese Academy of Sciences, Beijing 100049, China

3 Shandong Institutes for Food and Drug Control, Jinan 250100, Shandong, China 